\title{
Time of day influences immune response to an inactivated vaccine against SARS-CoV-2
}

\author{
(c) CEMCS, CAS 2021
}

Cell Research (2021) 31:1215-1217; https://doi.org/10.1038/s41422-021-00541-6

Dear Editor,

The coronavirus disease 2019 (COVID-19) pandemic that caused by severe acute respiratory syndrome coronavirus 2 (SARS-CoV-2) has affected millions of people and is an ongoing issue globally. The application of effective and safe vaccines is critical to achieving control of the pandemic. ${ }^{1}$ Data from clinical trials have shown that several vaccines can induce humoral and cellular immune responses against SARS-CoV-2. ${ }^{2-4}$ However, given the long time required for vaccine production, difficulty in vaccine distribution and public skepticism about their safety and benefits, the vaccine coverage rate is growing slowly worldwide. Therefore, to make full use of available resources and maximize the efficacy of vaccination is critically important in fighting against COVID-19.

Currently, there are limited data on the immune responses to SARS-CoV-2 vaccination regarding the potential impact of timing of vaccination in a 24-hour circadian rhythm cycle. We know that the immune system is influenced by circadian rhythm, and immune cells vary in cell number and immune function in the blood in a circadian fashion. ${ }^{5}$ In certain cases, antibody response to flu vaccines is stronger when vaccinated in the morning than in the afternoon. ${ }^{6}$ However, whether circadian rhythm affects immune response to SARS-CoV-2 vaccines is not known. We have therefore investigated the immunological response to SARS-CoV-2 vaccine delivered in the morning vs afternoon among healthcare workers (HCWs). The receptor binding domain (RBD) of SARS-CoV2 spike protein-based chemiluminescent immunoassay (CLIA) was utilized for neutralizing antibody (NAb) measurement, and flow cytometry for immune phenotype monitoring and antigenspecific memory B cell measurement.

To investigate the potential impact of vaccine delivery timing on immune responses, ${ }^{7}$ a prospective cohort study design was adopted. Sixty-three HCWs from the First Affiliated Hospital of Sun Yat-sen University (FAH-SYSU) in Guangzhou, China, received vaccination in the morning ( $9 \mathrm{am}-11 \mathrm{am})$ or afternoon (15 pm-17 pm) according to the assignment of relevant hospital staffs (Supplementary information, Table S1). All participants received an inactivated SARS-CoV-2 vaccine (BBIBP-CorV, Sinopharm, Beijing) on day 0 and day 28. The two doses of vaccine were given at the same time of a day. Blood samples were collected on day 0 , day 14 , day 21 , day 28 and day 56 . The study was approved by the research committee of FAH-SYSU and registered to Chinese Clinical Trial Registry (ChiCTR2100042222). Written informed consents were obtained from all participants. During the study, no serious adverse events related to the vaccination were recorded (Supplementary information, Table S1).

We found that the inactivated vaccine elicited strong immune responses. Percentages of antibody-secreting cells (ASCs) among $B$ cells and follicular helper $T$ (Tfh) cells were increased in the peripheral blood after vaccination (Fig. 1a). The kinetic changes of immune cell subsets in the peripheral blood revealed that there was an expansion of Tfh cells along with increased HLA-DR expression on $B$ cells, indicating a germinal center response was initiated by the vaccination. Other $\mathrm{T}$ cell subsets were largely unaffected by the vaccination (Supplementary information, Figs. S1, S2).

We monitored the dynamic changes of B cell subsets by flow cytometry (Supplementary information, Figs. S3, S4), and no significant changes of $B$ cells in the total naïve and memory compartments after the vaccination were observed (Supplementary information, Fig. S4b, c). As for the memory B cell subpopulations, we found that the percentages of switched memory, $\operatorname{lgD}^{+}$memory and unswitched memory $\mathrm{B}$ cells were not affected by the vaccination (Supplementary information, Fig. $\mathrm{S} 4 \mathrm{e}, \mathrm{g}, \mathrm{h}$ ), except that the percentage of $\operatorname{lgM}^{+}$memory $\mathrm{B}$ cells was increased (Supplementary information, Fig. S4f). It has been reported that extrafollicular $B$ cell response was enhanced in COVID-19 patients. The expansion of double negative (DN, $\left.\lg \mathrm{D}^{-} \mathrm{CD} 27^{-}\right) \quad \mathrm{B}$ cells, especially the DN2 $\left(\operatorname{lgD}{ }^{-} \mathrm{CD} 27^{-} \mathrm{CD} 11 \mathrm{c}^{+} \mathrm{CXCR5^{- }}\right)$ subset, was correlated with the neutralizing antibodies against SARS-CoV-2 in these patients. ${ }^{8}$ Here we found that the percentage of DN B cells was also increased after vaccination (Supplementary information, Fig. S4i). Unlike DN2 B cells, there was a robust induction of DN1 $\left(\operatorname{lgD} D^{-} \mathrm{CD} 27^{-} \mathrm{CD} 11 \mathrm{c}^{-} \mathrm{CXCR5^{+ } )}\right.$ subset by the inactivated vaccine (Supplementary information, Figs. S4j-I and S5), implying that B cells responded differently between pathological condition and vaccination. As for cytokine reaction, TNF-a was stimulated by the vaccination and maintained at a relatively high level when compared to the baseline. IL- 6 was increased after first dose, but retrieved to the baseline rapidly, while IL-10 was unaffected (Supplementary information, Fig. S6).

Further, serological response was shown to be induced by the vaccination and the concentration of NAbs was increased gradually after the first dose of vaccine. Although the concentration of NAbs was low after the first dose of vaccine, it was boosted strongly by the second dose (Fig. 1b). NAbs in the sera increased from $6.9 \mathrm{AU} / \mathrm{mL}$ at day 28 to $24.5 \mathrm{AU} / \mathrm{mL}$ at day 56 (Fig. 1b). Strikingly, participants vaccinated in the morning showed significantly higher level of NAbs in the sera, 34.70 (IQR: 21.61, 43.59) vs 19.35 (IQR: $11.83,25.09)$, and the difference with its $95 \%$ confidence interval is $14.84(7.37,24.15)$ (Fig. 1b, c). Participants vaccinated in the morning also experienced stronger $B$ cell and Tfh responses to the vaccination. By day 56, the percentages of ASC cells and $\mathrm{CD}_{138^{+}}$ASC cells were significantly higher in the morning vaccination group (Fig. 1d, e). The percentage of Tfh cells in the morning vaccination group was 1.4 fold to that of afternoon vaccination group (Fig. 1f, g). The percentages of monocytes and dendritic cells were also higher in the morning vaccination group 


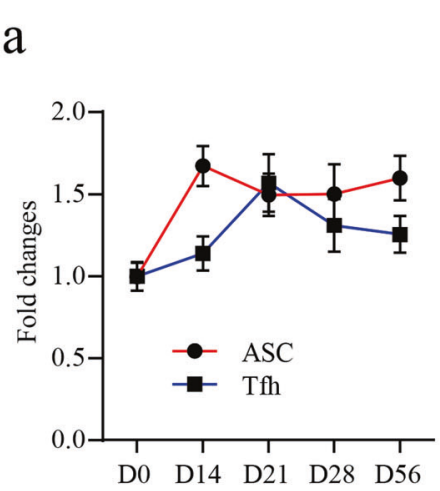

d

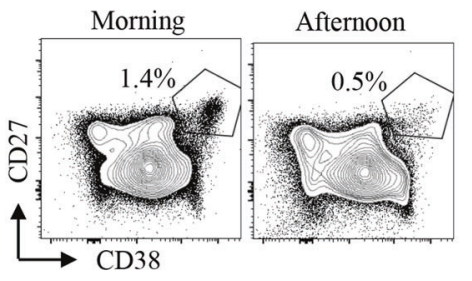

b

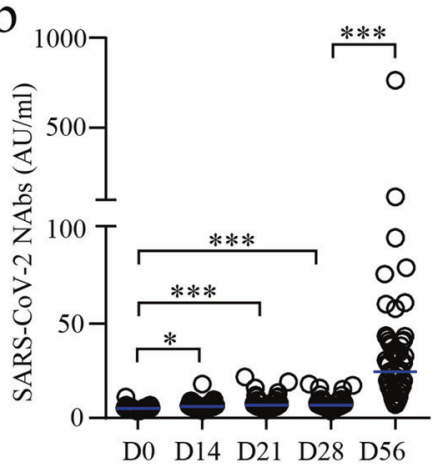

C

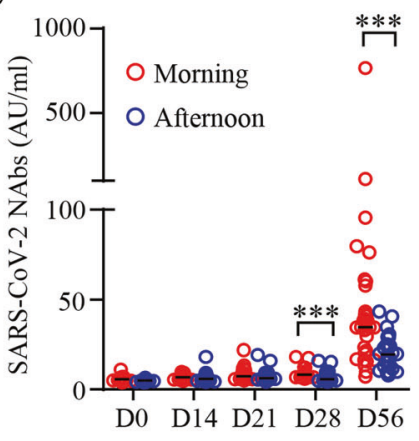

ASC

$\mathrm{CD} 138^{+} \mathrm{ASC}$

e

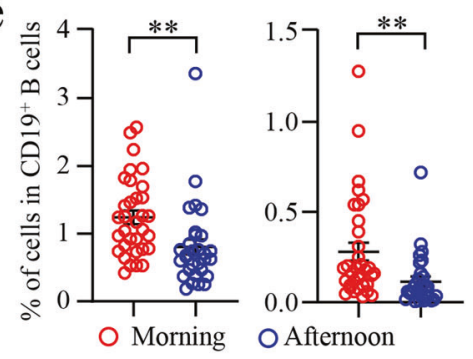

f

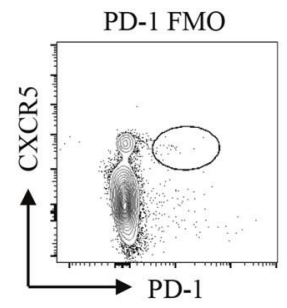

$\mathrm{h}$

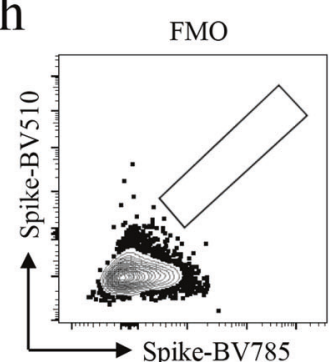

j

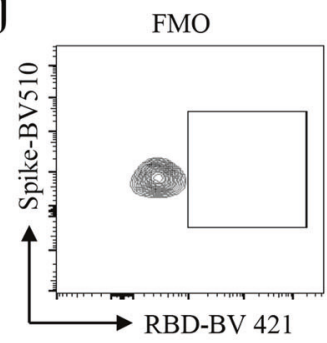

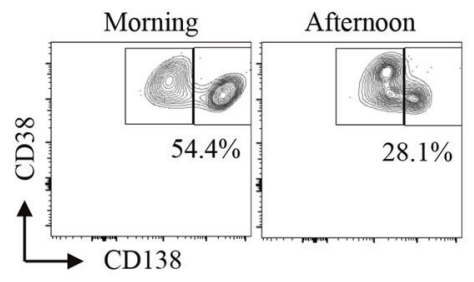

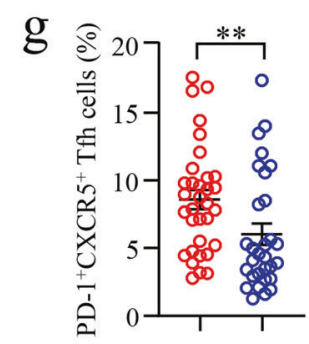

i
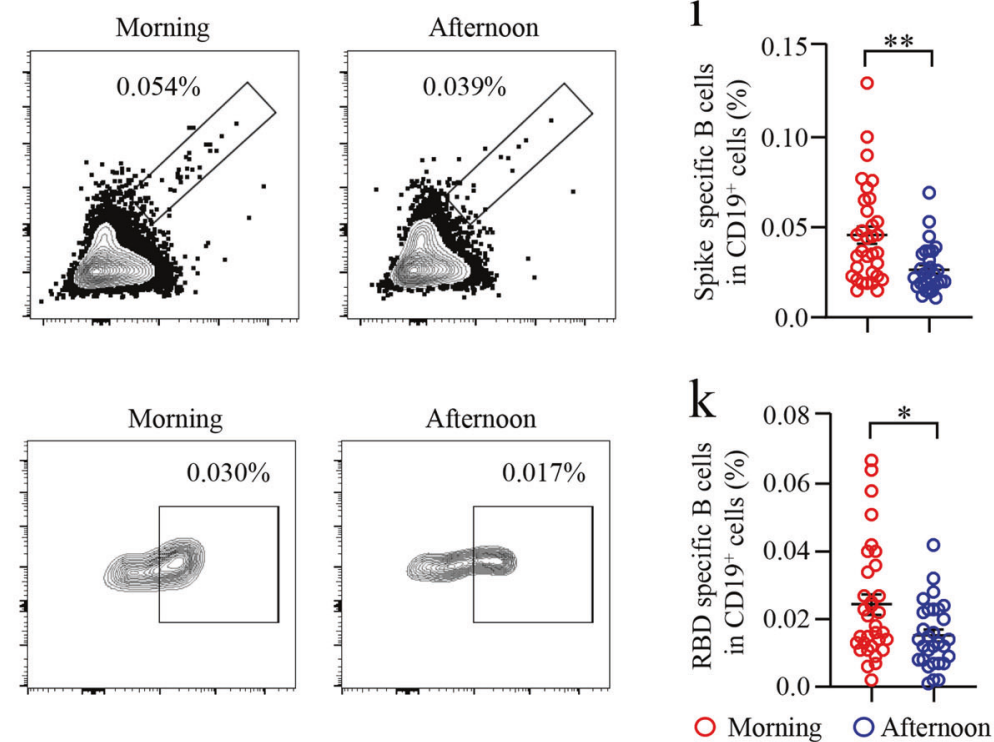

by day 56 (Supplementary information, Fig. S7). However, the extrafollicular response was not different between morning and afternoon vaccination (Supplementary information, Fig. S8).

Previous work shows that immunological memory to SARS-CoV2 is relative stable for up to 8 months after SARS-CoV- 2 infection.
Spike-specific memory B cells were even more abundant at 6 months than 1 month after symptom onset. ${ }^{9}$ Immunological memory is critical for durable protective immunity after vaccination. To further investigate whether there is an immune memory established after the vaccination, we analyzed SARS-CoV-2-specific 
Fig. 1 Stronger immune response by morning vaccination of an inactivated SARS-CoV-2 vaccine. The 63 healthcare workers (HCWs) who either received vaccination in the morning (9 am-11 am, $n=33)$ or afternoon (15 pm-17 pm, $n=30)$ on D0 and D28. a Blood samples from D0, D14, D21, D28 and D56 were stained with antibodies against CD3, CD4, CD19, CD27, CD38, CD138, PD-1 and CXCR5. Cells were then analyzed by flow cytometry for antibody-secreting cells (ASC): $\mathrm{CD} 3^{-} \mathrm{CD} 19^{+} \mathrm{CD} 27^{+} \mathrm{CD} 38^{++}$and follicular helper $\mathrm{T}$ cells (Tfh): $\mathrm{CD}^{+} \mathrm{CD} 4^{+} \mathrm{CD} 19^{-} \mathrm{PD} 1^{+} \mathrm{CXCR}^{+}$. Relative fold changes are shown. Data are means $\pm \mathrm{SEM}$. b, c NAbs against the RBD of SARS-CoV-2 spike protein in the sera were measured by chemiluminescent immunoassay (CLIA). Medians of the data are shown. Comparisons were done by Mann-Whitney $U$ test and Wilcoxon rank sum test. $\mathbf{d}-\mathbf{g}$ Blood samples collected on D56 were analyzed by flow cytometry. Gating strategy was performed as described above (d, f). Percentages of ASCs, CD138 ${ }^{+}$ASCs and Tfh cells were summarized and representative counter plots are shown $(\mathbf{e}, \mathbf{g})$. FMO, Fluorescence Minus One. h-k Blood samples from D56 were analyzed for spike- and RBD-specific memory B cells by flow cytometry, and representative counter plots were shown $(\mathbf{h}, \mathbf{j})$. Percentages of spike- and RBD-specific memory B cells were summarized for the morning and afternoon groups (i, k). Data are means \pm SEM. Comparisons were done by Student's $t$-test. ${ }^{*} P<0.05,{ }^{* *} P<0.01,{ }^{* * *} P<0.001$.

memory B cells in the peripheral blood of the vaccinated HCWs by flow cytometry, uncovering spike- and RBD-specific memory B cells in the blood (Supplementary information, Fig. S9). The percentages of spike- and RBD-specific memory B cells were 3.3 and 1.6 fold to that of afternoon vaccination group, respectively (Fig. 1h-k).

Our results demonstrate that the inactivated vaccine could stimulate strong serological response in HCWs, who also acquired long-term protective immunological memory against SARS-CoV-2. The establishment of immune memory against SARS-CoV-2 is critical for vaccine efficacy and long-term protection. Interestingly, our data suggest that vaccination in the morning leads to a stronger immune response to the inactivated vaccine than in the afternoon.

The immune system is influenced by circadian rhythm, and immune response varies at different times of day. ${ }^{10}$ Consistently, we also observed that immune cells in the blood changed from morning to afternoon (Supplementary information, Figs. S10, S11). Therefore, by taking advantage of the circadian rhythm of human immune system, stronger immune responses could be achieved by morning vaccination. Our study provides evidence that morning vaccination might enhance serological response and could therefore enhance the efficacy of vaccination in HCWs. Future studies are needed to validate these findings. Besides, if circadian rhythm does impact the immune responses, it is important to understand whether these variations are clinically significant and the potential changes that might be warranted for the current vaccination protocols, procedures, and/or dosages.

Hui Zhang ${ }^{1,2}$, Yihao Liu ${ }^{1,2}$, Dayue Liu ${ }^{1,2}$, Qin Zeng ${ }^{1,2}$, Liubing $\mathrm{Li}^{1,2}$, Qian Zhou ${ }^{1,2}$, Mengyuan $\mathrm{Li}^{1,2}$, Jie $\mathrm{Mei}^{1}$, Niansheng Yang (ID ${ }^{{ }^{\prime}}$, Suilin Mo', Qiusheng Liu', Min Liu', Sui Peng ${ }^{1,2}$ and Haipeng Xiao iD ${ }^{1,2}$

${ }^{1}$ The First Affiliated Hospital of Sun Yat-sen University, Guangzhou, Guangdong, China. ${ }^{2}$ These authors contributed equally: Hui Zhang, Yihao Liu, Dayue Liu, Qin Zeng, Liubing Li, Qian Zhou, Mengyuan Li, Sui Peng, Haipeng Xiao. ${ }^{\varpi}$ email: xiaohp@mail.sysu.edu.cn

\section{REFERENCES}

1. Jeyanathan, M. et al. Nat. Rev. Immunol. 20,615-632 (2020).

2. Sadoff, J. et al. N. Engl. J. Med. 384, 1824-1835 (2021).

3. Xia, S. et al. Lancet Infect. Dis. 21, 39-51 (2021).

4. Xia, S. et al. JAMA 324, 951-960 (2020).

5. Scheiermann, C. Nat. Rev. Immunol. 18, 423-437 (2018).

6. Long, J. E. Vaccine 34, 2679-2685 (2016).

7. de Bree, L. C. J. et al. J. Clin. Invest. 130, 5603-5617 (2020).

8. Woodruff, M. C. et al. Nat. Immunol. 21, 1506-1516 (2020).

9. Dan, J. M. et al. Science 371, eabf4063 (2021).

10. Druzd, D. et al. Immunity 46, 120-132 (2017).

\section{ACKNOWLEDGEMENTS}

We thank Drs. Ji Wang and Liping Wu for blood sample collection and technical support. This work was supported by The Talent Program of First Affiliated Hospital, Sun Yat-sen University (Y70311). H.Z. was supported by The Hundred Talent Program of Sun Yat-sen University (Y61229).

\section{AUTHOR CONTRIBUTIONS}

H.X. and S.P. supervised the study. H.X., S.P., H.Z. and Y.L. conceived and designed the study. Y.L., Q.Zeng, L.L., M.L. and J.M. collected and processed blood samples. Q.Zeng, M.L. and L.L. performed the experiments and collected data. Q.Zeng and Q.Zhou performed statistical analysis. H.Z., Y.L., D.L., Q.Zeng, L.L. and M.L. drafted the report. H.X., S.P., N.Y., S.M., Q.L. and M.L. made critical revision on the manuscript. All authors contributed to the interpretation of data, and revised the manuscript and approved the final version before submission.

\section{COMPETING INTERESTS}

The authors declare no competing interests.

\section{ADDITIONAL INFORMATION}

Supplementary information The online version contains supplementary material available at https://doi.org/10.1038/s41422-021-00541-6.

Correspondence and requests for materials should be addressed to H.X.

Reprints and permission information is available at http://www.nature.com/reprints 